\title{
Appendix insulin secreting neuroendocrine tumor in a diabetic patient: a challenging diagnosis
}

\author{
Lombardi $\mathbf{M}^{1 *}$, Battezzati $\mathbf{M A}^{2}$, Grosso $\mathrm{F}^{3}$, Muni $\mathrm{A}^{4}$, Volante $\mathbf{M}^{5}$, Ansaldi $\mathrm{E}^{2}$ \\ ${ }^{1}$ Internal Medicine Department, Versilia Hospital, USL Toscana Nordovest, Italy \\ ${ }^{2}$ Endocrinology Department, SS. Antonio and Biagio and Cesare Arrigo Hospital, Alessandria, Italy \\ ${ }^{3}$ Oncology Department, SS. Antonio and Biagio and Cesare Arrigo Hospital, *Italian Rare Cancer Network, Alessandria, Italy \\ ${ }^{4}$ Nuclear Medicine Department, SS. Antonio and Biagio and Cesare Arrigo Hospital, Alessandria, Italy \\ ${ }^{5}$ Department of Oncology, Pathology Unit, University of Turin, San Luigi Hospital, *Italian Rare Cancer Network, Italy
}

Received: September 7, 2016; Accepted: November 10, 2016; Published: January 2, 2017

*Corresponding author: Martina Lombardi, MD, Internal Medicine Department, St Antonio Abate Hospital, Pontremoli, USL Toscana Nordovest, Via Nazionale 16, 54027, Pontremoli, Massa Carrara, Italy; Tel: +39 0187-462248; Fax:+39 01874-62247; E-mail: martina.lombardi@uslnordovest.toscana.it

\begin{abstract}
Context: Tumor-induced hypoglycemia is a rare entity and it is mainly due to pancreatic insulinomas. Non-islet cell tumor hypoglycemia is really exceptional and ectopic insulin secretion has been previously suggested.
\end{abstract}

Case description: A 79 year-old type 2 diabetic man, after over 30 years of poorly controlled diabetes, observed an unexplained improving of glycemic control with recurrent hypoglycemia. He progressively reduced insulin doses, till suspension, with persistent frequent hypoglycemia. A fasting test documented symptomatic hypoglycemia with inappropriate elevated insulin and C-peptide. CT scan and endoscopic ultrasound did not reveal any pancreatic lesion. A 68Gallium-DOTANOC showed a focal pathological right pelvic uptake, corresponding to an oval enhancing lesion at the targeted CT images. The patient was submitted to surgical excision of that mass, revealing an appendix neuroendocrine well differentiated tumor with lymph nodes metastasis, showing partial insulin immunohistochemistry staining. After surgery no other hypoglycemic events were documented; to control diabetes insulin therapy needed to be reintroduced.

Conclusion: Extra-pancreatic insulin secreting tumors are very rare and their diagnosis in diabetic patients can be challenging. This case addresses the diagnosis and treatment of this rare entity reporting, to our knowledge, the first case of ectopic insulinoma, arising from appendix.

Key words: Ectopic insulin secretion neuroendocrine tumor; Hypoglycemia; Diabetes.

\section{Introduction}

The most frequent cause of hypoglycemia is a side effect of insulin or other anti diabetic drugs overdose.

In non diabetic subjects, hypoglycemia is usually due to endogenous insulin hyperproduction, coming from nesidioblastosis or insulin secreting pancreatic tumors, arising from islet $\beta$ cells. On other rare occasions, hypoglycemia can occur as a consequence of auto-antibodies towards insulin or its receptor that are mainly associated with para-neoplastic syndromes [1].

Tumor induced hypoglycemia (TIH) can rarely be developed by extra-pancreatic tumors and it can be due to insulin ectopic secretion or to other neuroendocrine pathogenic mechanisms such as IGF2, IGF1, somatostatin or GLP1 overproduction [2].

$\mathrm{TIH}$, due to ectopic insulin secretion, usually results from duodenum or peri-pancreatic tumors. The majority of these rare tumors come from ectopic accessory or aberrant pancreas [3]. Anyway, anecdotal cases reported extra pancreatic insulin secreting tumors such as kidney neuroendocrine tumor, bronchial carcinoid, cervix carcinomas, paraganglioma, schwannomma, and gastrointestinal tumor [2].

Herein we report, to our knowledge, the first case of well documented appendix well differentiated neuroendocrine insulin secreting tumor.

\section{Case-report}

A 79-year old man, diagnosed with diabetes in 1980 was treated with insulin for many years, with poor glycemic control. Suddenly, he developed recurrent hypoglycemic episodes. Any relevant weight, diet or activities changes were reported. As suggested by his diabetologist, the patient gradually reduced, till suspension, the dose of insulin therapy, with persistent recurrent hypoglycemias. After two weeks, since insulin interruption, basal fast serum glycemia was $53 \mathrm{mg} / \mathrm{dl}$, insulin $99 \mathrm{mUI} / \mathrm{ml}$, C-peptide $10 \mathrm{ng} / \mathrm{ml}$ and Chromogranin A (without proton pump inhibitor or other eventually interfering drugs) $435 \mathrm{ng} / \mathrm{ml}$ (Normal value $0-100 \mathrm{ng} / \mathrm{ml}$ ). Thyroid function, serum cortisol and cathecolomines were normal.

A 72-h fasting test was started; it was stopped after $24 \mathrm{~h}$ for a symptomatic hypoglycemia (glycemia $38 \mathrm{mg} / \mathrm{dl}$ ) associated with inappropriate elevated insulin and C-peptide (Insulin $47.8 \mathrm{mUI}$ / 
$\mathrm{ml}, \mathrm{C}$-peptide $4,4 \mathrm{ng} / \mathrm{ml}$ ) and with a pathological response to glucagon (glycemia increasing from 38 to $90 \mathrm{mg} / \mathrm{dl}$ at 90 minutes after glucagon infusion).

The patient was submitted to contrast enhanced abdominal CT and to endoscopic ultrasound, without any documented pancreatic lesions. However, subsequent 68Gallium-DOTANOC (Figure 1) showed a focal pathological uptake in the right pelvic region that corresponded to an oval pelvic mass, at the following targeted CT images.

He was submitted to abdominal laparoscopic surgery that showed a caecal appendix mass that was removed together with local lymph nodes. Pathology examination found an appendix of $5.5 \mathrm{~cm}$ length with a diameter of $0.4-1.4 \mathrm{~cm}$, surrounded by congested serosa. Appendix lumen was occupied by a whitish necrotic lesion, infiltrating the appendix wall but not the peri-appendix tissue. Microscopically it was suggestive of a neuroendocrine appendix grading 2 tumor, with necrosis, infiltrating the appendix wall till subserosa, with lymphatic and vascular invasion and metastasis of one local lymph node (pT2N1). Immunohistochemical staining revealed diffuse and intense staining for chromogranin, synaptophysin and partial positivity for insulin. MIB1 labeling index was $10 \%$ and mitotic rate was 2 per 10 high power fields (Figure 2). The case was share in the Italian Rare Cancer Network to obtain an expert pathological confirmation.

After surgery the patient did not presented any other hypoglycemic episodes and, due to relapsed stable hyperglycemia, he reintroduced basal-bolus insulin treatment. Two months after surgery serum chromogranin A was normalized without any other specific treatment. He will continue appropriate morphological and biochemical follow up, to exclude any neoplastic recurrence.

\section{Discussion}

Diabetic patients, especially if treated with insulin, are at high risk of hypoglycemia. In diabetic patient, any hypoglycemic event is generally ascribed to hypoglycemic therapy, thus the diagnosis of insulinoma is more complex.

Incidence of diabetes in patients with insulinoma is lower than in general population [4] and there are only sporadic case reports of insulinoma in previously diabetic patients [5].

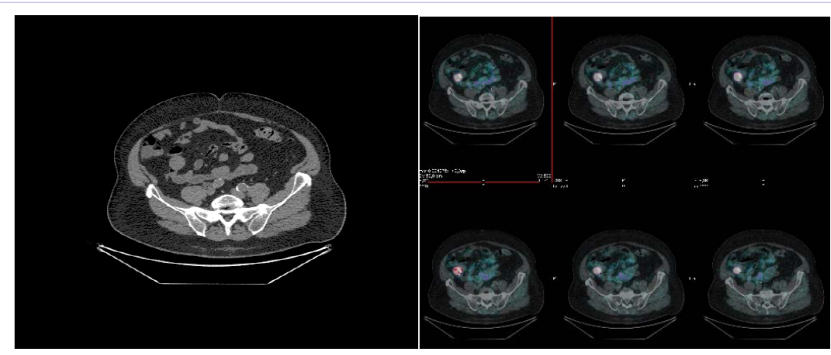

Figure 1: Gallium-68 DOTANOC images showing focal pathological uptake in the right pelvic region, corresponding to an oval pelvic mass, at the targeted CT images
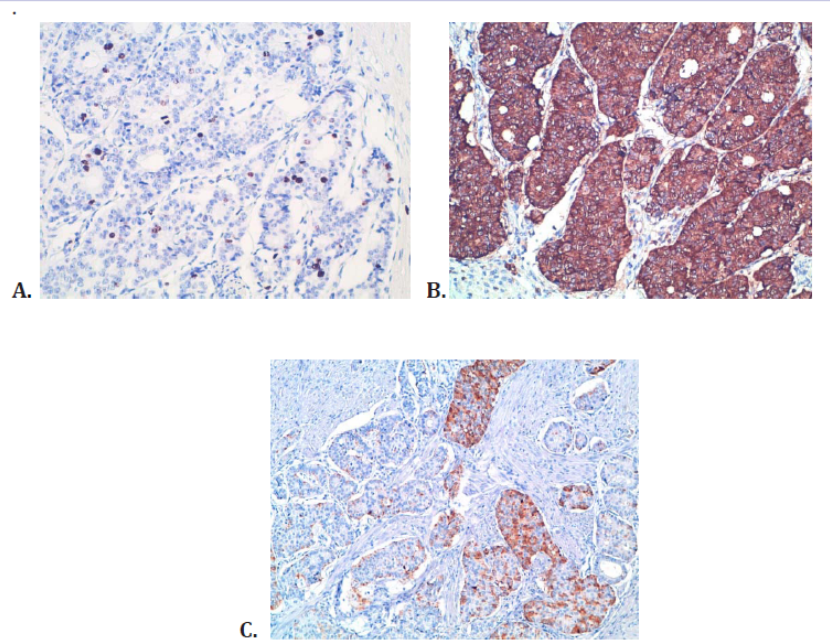

Figure 2: A. Immunohistochemistry for Ki67, B. Chromogranin A, C. Insulin

However, when hypoglycemia persists after suspension of the hypoglycemic treatment, an insulinoma must be suspected.

Biochemical diagnosis of hypoglycemia is established when a Whipple's triad is documentated (signs or symptoms of hypoglycemia, low plasma glucose concentration $(<40$ $\mathrm{mg} / \mathrm{dl}$ ) and resolution of signs and symptoms after glucose somministration). Hypoglycemia can then be ascribed to insulinoma if a spontaneous hypoglycemia is associated to concomitant insulin levels $\geq 6 \mu \mathrm{U} / \mathrm{ml}$ and C-peptide levels $\geq 200$ $\mathrm{pmol} / \mathrm{l}$ (or proinsulin levels $\geq 5 \mathrm{pmol} / \mathrm{l}$ ), with the absence of sulfonylurea in the plasma or urine [6]. Alternatively, 72-h fasting test should be organized to document a fasting hypoglycemia with concomitant inappropriate elevated insulin and C-peptide and/or proinsulin levels [6].

At the end of $72 \mathrm{~h}$-fasting tests, other useful diagnostic tools, predictive of insulinoma are: beta-hydroxybutyrate less than 2.7 $\mathrm{mmol} / \mathrm{l}$ and a glucose response to $1 \mathrm{mg}$ glucagon IV greater than $25 \mathrm{mg} / \mathrm{dl}$ [7].

In this case we documented a persistent recurrent hypoglycemia in a patient with previously poorly controlled long term diabetes, although the patient did not refer any life style or weight changes. Elevated basal insulin, C- peptide and chromogranin A were suspected for an insulinoma. This hypothesis was then confirmed after the $72 \mathrm{~h}$ fasting test during which a clear hypoglycemia was documented and associated to insulin and C-peptide in the range suggestive of insulinoma. Moreover, after glucagone test, glycemia increased of $52 \mathrm{mg} / \mathrm{dl}$, corroborating the idea of insulinoma.

Insulinomas are almost universally located within the pancreas $(1 / 3$ head $-1 / 3$ body $-1 / 3$ tail), although they are often so small that their pre-surgical localization could become very difficult. As first line radiological assessment, insulinomas are generally investigated through abdominal ultrasound, CT or 
MRI [7]. Endoscopic ultrasound can then help in localizing the pancreatic tumor, with a positive imaging in $70-95 \%$ of cases.

Althoght only about $20-50 \%$ of insulinomas express somatostatin receptors, an Octreoscan ${ }^{\circledR}$ or, more frequently, 68Gallium-DOTANOC or DOTANOC can be useful to localize the tumor [8]. Moreover, new nuclear imaging methods are recently been proposed, such as scintigraphy using radio labeled GLP-1 analogues, that seems to be really promising in presurgical insulinoma localization. When all the morphological investigations remain negative, selective arterial calcium stimulation with hepatic venous sampling for insulin, can help in localizing the tumor within the pancreatic head, corpus or tail, before proceeding to an eventual surgical exploration, guided by pancreatic manual palpation and intra-operative ultrasound.

In the reported case, abdominal CT and endoscopic ultrasound did not find any pancreatic lesion. However, 68Gallium-DOTANOC showed a pathological uptake in the right pelvic region, where the CT images denoted an ovoid mass.

Hypoglycemia induced by non pancreatic tumor is a very rare entity and its pathogenesis is not easily identified. Different mechanisms have been previously proposed: (a) extrapancreatic insulinoma; (b) ectopic insulin production; (c) increased consumption of glucose by a tumor; (d) ectopic production of insulin-like peptide (IGF2, IGF1) or GLP1.

Extra pancreatic insulinoma is an extremely infrequent condition, reported in only $1-2 \%$ of all insulinomas and it has to be suspected when a biochemically confirmed insulinoma cannot be localized with radiological images. These very rare tumors have been mainly reported to develop from ectopic pancreas, generally observed in the duodenal wall or in the peripancreatic tissues [9]. Another quite rare event is a non-islet cell tumor, causing an ectopic insulin secretion such as it has been reported for some bronchial carcinoids, kidney neuroendocrine tumor, paragangliomas, cervix carcinoma, shwannoma and gastrointestinal stromal tumors.

In this case we are reporting a case of appendix neuroendocrine, grade 2 tumors, with an insulin positive immunohistochemistry. This is to our knowledge, the first case of an appendix insulin-secreting neuroendocrine tumor. We could only find a case report of an appendix carcinoid with liver metastasis with reported signs of hyperinsulinemia and a weak and poorly expressed autoptical immunohistochemistry reaction for insulin. However, in this previously described case, a real hypoglycemia and a biochemical diagnosis of insulinoma could not be done [10].

Surgery is the first line treatment for insulin secreting neuroendocrine tumor, trying to perform a surgical resection, guided from a radiological pre-operative localization or trying to find it out with a surgical exploration.

This reported case underlines how an accurate pre-surgical evaluation can identify even a really unusual or unique source of ectopic insulin secretion, obtaining a complete control of hypoglycemia and maybe a better prognosis. Prognosis of these neuroendocrine tumors mainly depends upon the grade and stage of the disease. Thanks to a quite prompt diagnosis, after the beginning of symptoms, the grade 2 neuroendocrine tumor was diagnosed before local and distant metastasis could become clinically evident, permitting a likely radical surgery with a possible complete and lastly neoplastic remission. Whenever a clinical insulinoma is diagnosed, if localization with traditional and new imaging cannot be obtained, a possible ectopic insulin secretion should be supposed, trying to rule it out with the best now available techniques such as Gallium-DOTATOC/DOTANOC or radiolabeled GLP-1 analogues, in order to improve the patient prognosis.

\section{References}

1. Cryer PR, Axelrod L, Grossman AB, Heller SR, Montori VM, Seaquist ER, et al. Evaluation and managenement of adult hypoglycemic disorders: an Endocrine Society Clinical Practice Guideline. J Clin Endocrinol Metab. 2009;94(3):709-728.

2. Iglesias P, Diez J. Management of endocrine disease: A clinical update on tumor-induced hypoglycemia. Eur J Endocrinol. 2014;170(4):147157.

3. Hennings J, Garske U, Botling J and Hellman P. Malignant insulinoma in ectopic pancreatic tissue. Dig Surg. 2005;22(5):377-379.

4. Levine RA, Sobel BE. Insulinoma, type 2 diabetes and plasminogen activator inhibitor type-I. Coron artery Dis. 2001;12(4):333-336.

5. Hameed mf, Hoyle G, Muir Z. A mysterious case of normalising blood sugar: insulinoma in a long-standing diabetic patient. Age and ageing. 2006;35(3):317-318. doi: 10.1093/ageing/afj089.

6. Jensen RT, Cadiot G, Brandi ML, de Herder WW, Kaltsas G, Komminoth $\mathrm{P}$, et al. ENETS Consensus Guidelines for the Management of Patients with Digestive Neuroendocrine Neoplasms: Functional Pancreatic Endocrine Tumor Syndromes. Neuroendocrinology. 2012;95(2):98119. doi: $10.1159 / 000335591$.

7. O’ Brien T, O’ Brien PC, Service FJ. Insulin surrogates in insulinoma. J Clin Endocrinol Metab. 1993;77(2):448-451.

8. Sundin A, Garske U, Orlefors H. Nuclear imaging of neuroendocrine tumours. Best Pract Res Clin Endocrinol Metab. 2007;21(1):69-85.

9. Hennings J, Garske U, Botling J and Hellman P. Malignant insulinoma in ectopic pancreatic tissue. Dig Surg 2005;22(5):377-379.

10. Urbanczyk K, Tomaszewska R. Cytological pattern of multihormonal carcinoid of the appendix with metastasis to the liver corresponding a clinical insulinoma. Pat Pol. 1994;45(3):225-229. 